\title{
The added advantage of automated breast ultrasound to mammographically detected different breast lesions in patients with dense breasts
}

\author{
Engy A. Ali', Alaa M. Ahmed and Noha A. Elsaid
}

\begin{abstract}
Background: Breast cancer is the most commonly diagnosed malignancy in women worldwide. Women with dense breast tend to have 15-25\% lifetime risk of breast cancer due to decrease of mammographic sensitivity. Automated breast ultrasound (ABUS) is a new promising tool for detection of breast lesions masked by dense glandular tissue at mammography.

Results: The sensitivity of digital mammography in detecting breast lesions was $60.7 \%$, specificity $91.6 \%$, PPV $85 \%$, NPV $75 \%$, and accuracy $78 \%$. The sensitivity of ABUS in detecting breast lesions was $92.86 \%$, specificity $77.78 \%$, PPV 76.47\%, NPV 93.33\%, and accuracy $84.38 \%$. The sensitivity of handheld ultrasound (HHUS) in detecting breast lesions was $89.29 \%$, specificity $88.89 \%$, PPV $86.21 \%$, NPV $91.43 \%$, and accuracy $89.06 \%$.

Conclusion: The sensitivity of ABUS in detecting breast lesions was much higher than mammography in dense breast while the digital mammography (DM) had higher specificity. So, implementation of both DM and ABUS to get benefit of DM specificity as well as ABUS sensitivity were highly recommended.
\end{abstract}

Keywords: Dense breast, Automated breast ultrasound

\section{Background}

Breast cancer is the leading cause of cancer-related death among females worldwide [1]. Early detection of breast cancer improves outcomes, i.e., survival is relatively good when these cancers are diagnosed at an early stage [2].

A recent study showed $43 \%$ number reduction among women participating in a national screening program [3].

Mammography is an effective randomized controlled trial-proven method for reducing mortality due to breast cancer [4].

The sensitivity of mammography depends on breast density. Studies on women with dense breasts have demonstrated a sensitivity of less than 50\% [5].

* Correspondence: drengyali78@gmail.com

Diagnostic and Intervention Radiology Department (Women's Imaging Unit, Cairo University Hospitals, Kasr Al-Ainy, El-Manial, Cairo 11956, Egypt
Women with extremely dense breasts also have a 4.7fold increased risk of developing breast cancer compared with women with fatty-replaced breasts. There is about 18 -fold increased risk of an interval cancer in women with dense breast tissue [6].

Cancers detected in women with dense breast tissue are larger and more frequently node positive [7].

The role of radiologists in imaging the breast is vital. At present, X-ray mammography is the "gold standard" for screening and early detection of breast cancer [8].

Women with dense breast tissue constitute the largest population of "intermediate risk" women-that is, women with a $15-25 \%$ lifetime risk of breast cancer. They have the "perfect storm" of decreased mammographic sensitivity and increased risk of breast cancer [9].

Breast ultrasonography (US) is currently considered the first-line examination in the detection and characterization

\section{Springer Open}

() The Author(s). 2020 Open Access This article is licensed under a Creative Commons Attribution 4.0 International License, which permits use, sharing, adaptation, distribution and reproduction in any medium or format, as long as you give appropriate credit to the original author(s) and the source, provide a link to the Creative Commons licence, and indicate if changes were made. The images or other third party material in this article are included in the article's Creative Commons licence, unless indicated otherwise in a credit line to the material. If material is not included in the article's Creative Commons licence and your intended use is not permitted by statutory regulation or exceeds the permitted use, you will need to obtain permission directly from the copyright holder. To view a copy of this licence, visit http://creativecommons.org/licenses/by/4.0/. 
of breast lesions. However, conventional handheld US (HHUS) has several limitations such as operator dependence and the requirement of a considerable amount of radiologist time for whole-breast US [10].

ABUS has several advantages over HHUS, such as higher reproducibility, less operator dependence, and less required physician time for image acquisition [11].

Recent studies have reported that ABUS is promising in US screening for women with dense breasts and can potentially replace handheld second-look US in a preoperative setting [10].

\section{Aim of work}

The aim of this study was to assess the ability of ABUS to detect mammographically occult breast lesions at dense breasts, assessing the diagnostic parameters of ABUS compared to digital mammography as well as HHUS in detection of breast lesions in dense breast. The secondary outcome was to prove the effectiveness of using ABUS as a screening tool in dense breasts in BIRADS 0 mammography results.

\section{Methods}

Prospective study was conducted on 59 patients presented with either palpable breast mass or as a part of early screening starting from January 2017 till July 2018. Their ages ranged from 24 to 81 years (mean age, $41 \pm$ 10 SD years). Three cases were represented with bilateral lesions and two cases had two lesions in the same breast.

\section{Inclusion criteria}

Dense breast (ACR C or ACR D) on digital mammography.

\section{Exclusion criteria}

Breasts with American College of Radiology (ACR) A (predominantly fatty breast) or ACR B (scattered glandular tissue) detected with digital mammography were excluded.

\section{Limitations of the ABUS in the study}

- Exclusion of axillary regions from the field of view.

- The absence of tools to assess vascularity and tissue elasticity.

- Artifacts that occur during data acquisition remain an issue that can cause false positive results or can obscure actual findings. The area where the most significant artifact usually occurs is in the subareolar region.

\section{Methods}

All of the cases $(n=59)$ were subjected to both digital mammography and automated breast ultrasound, as well as routine handheld ultrasound. They were asked to expose the upper part of the body. No other special preparations were needed.

a) Digital mammography examination protocol design

A craniocaudal (CC) and a medio-lateral oblique (MLO) views were obtained with the patient in a standing position. Breast compression was applied. Images were acquired with a mammography system Senographe Essential, GE Healthcare fullfield digital mammography machine. Senographe Essential has dual anode (rhodium molybdenum) with CsI digital detector.

b) Automated breast examination protocol design

All participants underwent ABUS examination. All ABUS exams were done with an ABUS system (Invenia TM ABUS, Automated Breast Ultrasound System, GE Healthcare, Sunnyvale, CA, USA) with high frequency probe. The transducer length is $15.3 \mathrm{~cm}$, with $6-15 \mathrm{MHz}$ frequency. The gray scale levels were 256 with frame rate 10 frames/second. The examination was performed in the supine position.

A cushion placed under the shoulder that helped to spread out the breast tissue evenly, with the nipple pointing to the ceiling. A hypoallergenic lotion placed evenly on the breast with an additional amount on the area of the nipple.

A disposal membrane was used to aid an acoustic coupling and one of the three levels of compression was applied to spread out the breast evenly with respect to image quality and patient comfort. The ABUS scan was continuous and automated. During the acquisition, women were asked not to move and to breathe smoothly.

Volume acquisitions were obtained in the axial plane starting from the inferior part of the breast with coronal and sagittal reconstruction.

Image data automatically acquired a $15.4 \mathrm{~cm} \times 17.0$ $\mathrm{cm}$ volume from the skin to the chest wall up to 5 $\mathrm{cm}$ deep with $0.2 \mathrm{~mm}$ thickness of each slice. For each breast, three volumes were obtained: the central (anteroposterior) volume with the nipple in the center of the footprint, the lateral volume that included the upper outer part of the breast tissue with the nipple located in the inferior-medial corner, and the medial volume that included the inner and inferior part of the breast tissue. A nipple marker was placed in every examination for the accurate co-ordinance. For optimal image quality, a selection between three breast 
Table 1 Correlation between mammography BIRADS and pathology

\begin{tabular}{|c|c|c|c|c|c|c|}
\hline & \multicolumn{5}{|c|}{ Final pathology } & \multirow[t]{3}{*}{$\boldsymbol{P}$ value } \\
\hline & \multicolumn{2}{|l|}{ Benign } & \multicolumn{3}{|c|}{ Malignant } & \\
\hline & & Count & $\%$ & Count & $\%$ & \\
\hline \multirow[t]{6}{*}{ Mammography BIRADS } & BIRADS 0 & 14 & 38.9 & 10 & 35.7 & \multirow[t]{6}{*}{$<0.001$} \\
\hline & BIRADS II & 1 & 2.8 & 0 & .0 & \\
\hline & BIRADS III & 18 & 50.0 & 1 & 3.6 & \\
\hline & BIRADS IV & 3 & 8.3 & 16 & 57.6 & \\
\hline & BIRADS V & 0 & .0 & 1 & 3.6 & \\
\hline & BIRADS VI & 0 & .0 & 0 & .0 & \\
\hline
\end{tabular}

sizes was made. In women with larger breasts additional views were taken to avoid tissue exclusion.

c) Handheld ultrasound images (HHUS)

The gel was applied to the breasts and ultrasound examination was done using radial and antiradial techniques with axilla, with probe frequency $18-5 \mathrm{MHz}$.

\section{Image analysis}

The digital mammography and automated ultrasound data were evaluated by two experienced radiologists in consensus; both observers were unaware of the pathological data of each patient.

\section{Digital mammography images}

Assessment of breast composition, mass characterization (shape, margin density), asymmetry, calcification, mass number, location, axillary lymphadenopathy, extension, skin thickening, retraction and architectural distortion, and BIRADS classification was done.

\section{Automated ultrasound images and handheld ultrasound images}

Assessment of mass characterization (shape, margin orientation, echopattern, posterior feature, calcification), mass number, location, axillary lymphadenopathy, skin thickening, retraction, and BIRADS classification were

Table 2 Percentage of different BIRADS at HHUS

\begin{tabular}{llll}
\hline & & Count & $\%$ \\
\hline HHUS BIRADS & BIRADS 0 & 1 & 1.6 \\
BIRADS I & 9 & 14.1 \\
BIRADS II & 3 & 4.7 \\
BIRADS III & 22 & 34.4 \\
BIRADS IV & 21 & 32.8 \\
BIRADS V & 8 & 12.5 \\
BIRADS VI & 0 & .0 \\
\hline
\end{tabular}

done. Additionally, for ABUS, we assessed lesions' character in coronal view.

All breast masses included in this study were interpreted as above described and then the accuracy in reaching the final diagnosis was calculated for digital mammography and automated ultrasound as well as HHUS.

Pathological results were used as the gold standard of reference for the 64 lesions apart from 13 lesions which were proven by HHUS criteria to be benign ( 9 diagnosed as fibroadenomas (follow up) and 4 as simple cysts).

Samples were obtained with fine needle aspiration cytology (FNAC), cytology of nipple discharge, core biopsy, surgical excision, and/or radical surgery. Analysis of the samples was performed in the pathology department by a group of well-trained expert pathologists.

\section{Statistical analysis}

Data were coded and entered using the statistical package SPSS (Statistical Package for the Social Sciences) version 25. Data were summarized using mean, standard deviation, median, minimum and maximum in quantitative data, and using frequency (count) and relative frequency (percentage) for categorical data.

Comparisons between quantitative variables were done using the non-parametric Mann-Whitney test. For comparing categorical data, chi square $\left(x^{2} 2\right)$ test was performed. Exact test was used instead when the expected frequency is less than 5 . Correlations between

Table 3 Percentage of different BIRADS at ABUS

\begin{tabular}{llll}
\hline & & Count & $\%$ \\
\hline ABUS BIRADS & BIRADS I & 7 & 11.1 \\
& BIRADS II & 2 & 3.2 \\
BIRADS III & 20 & 31.7 \\
BIRADS IV & 29 & 46.0 \\
BIRADS V & 5 & 7.9 \\
& BIRADS VI & 0 & .0 \\
\hline
\end{tabular}


Table 4 Comparison between statistics of mammography, ABUS, and HHUS

\begin{tabular}{llll}
\hline Statistic & Mammography & ABUS & HHUS \\
\hline Sensitivity & $60.7 \%$ & $92.86 \%$ & $89.29 \%$ \\
Specificity & $91.6 \%$ & $77.78 \%$ & $88.89 \%$ \\
Positive predictive value & $85 \%$ & $76.47 \%$ & $86.21 \%$ \\
Negative predictive value & $75 \%$ & $93.33 \%$ & $91.43 \%$ \\
Accuracy & $78 \%$ & $84.38 \%$ & $89.06 \%$ \\
\hline
\end{tabular}

quantitative variables were done using Spearman correlation coefficient. Standard diagnostic indices including sensitivity, specificity, positive predictive value (PPV), negative predictive value (NPV), and diagnostic efficacy were calculated. Testing for agreement between different methods in numerical data was done using the intra class coefficient (ICC) with $95 \%$ confidence interval (95\% CI). $P$ value less than 0.05 was considered as statistically significant.

\section{Results}

\section{Patient's demographics}

This prospective study included a total of 59 patients with 64 lesions presented with breast masses (detected by clinical examination or by mammography examination). Their ages ranged from 24 to 81 years (mean age, $41 \pm 10$ SD years).

Thirty-six lesions (56.2\%) were diagnosed as benign while 28 (43.8\%) lesions were diagnosed as malignant.

Pathological results were used as the gold standard of reference apart from 13 lesions which were proven by
HHUS criteria to be benign, 9 diagnosed as fibroadenomas (follow up) and 4 as simple cysts.

Each examination (digital mammography, ABUS, and HHUS) was evaluated regarding the following criteria according to the 5 th edition of BIRADS lexicon.

\section{Digital mammography}

As regards lesion detection 25 lesions (39.1\%) out of 64 lesions were not detected by mammography, 14 (56\%) of them were benign, and 11 (44\%) were malignant.

As regards breast density, 44 (68.8\%) of breasts examined were ACR C, while 20 (31.2\%) were ACR D. No significant correlation in our study between glandular tissue composition breast and malignancy $(P$ value 0.221$)$.

As regards the BIRADS evaluation of different lesions by mammography, 27 lesions were considered BIRADS 0 for further evaluation by other imaging tools, 1 lesion was considered BIRADS II, 19 lesions were considered BIRADS III, 16 lesions were considered BIRADSIV, and 1 lesion was considered BIRADS V (Table 1).

True positive $=17(60.7 \%)$, false positive $=3(8.3 \%)$, false negative $=11(39.3 \%)$, true negative $=33(91.7 \%)$

\section{HHUS}

As regards lesion detectability, it was higher than mammography, it could detect 15 lesions missed by mammography; however, it was lower than ABUS. ABUS could detect duct papilloma interpreted by HHUS

Table 5 Agreement between ABUS and HHUS BIRADS

\begin{tabular}{|c|c|c|c|c|c|c|c|c|c|c|c|c|c|c|c|c|}
\hline & & \multicolumn{14}{|c|}{ ABUS BIRADS } & \multirow[t]{3}{*}{$\boldsymbol{P}$ value } \\
\hline & & \multicolumn{2}{|c|}{ BIRADS I } & \multicolumn{2}{|c|}{ BIRADS ॥ } & \multicolumn{2}{|c|}{ BIRADS III } & \multicolumn{2}{|c|}{ BIRADS IV } & \multicolumn{2}{|c|}{ BIRADS V } & \multicolumn{2}{|c|}{ BIRADS VI } & \multicolumn{2}{|c|}{ BIRADS 0} & \\
\hline & & Count & $\%$ & Count & $\%$ & Count & $\%$ & Count & $\%$ & Count & $\%$ & Count & $\overline{\%}$ & Count & $\overline{\%}$ & \\
\hline \multirow[t]{7}{*}{ HHUS BIRADS } & BIRADS I & 8 & 100.0 & 0 & .0 & 1 & 5.0 & 0 & .0 & 0 & .0 & 0 & .0 & 0 & .0 & \multirow[t]{7}{*}{$<0.001$} \\
\hline & BIRADS II & 0 & .0 & 2 & 100.0 & 1 & 5.0 & 0 & .0 & 0 & .0 & 0 & .0 & 0 & .0 & \\
\hline & BIRADS III & 0 & .0 & 0 & .0 & 17 & 85.0 & 5 & 17.2 & 0 & .0 & 0 & .0 & 0 & .0 & \\
\hline & BIRADS IV & 0 & .0 & 0 & .0 & 1 & 5.0 & 19 & 65.5 & 1 & 20.0 & 0 & .0 & 0 & .0 & \\
\hline & BIRADS V & 0 & .0 & 0 & .0 & 0 & .0 & 4 & 13.8 & 4 & 80.0 & 0 & .0 & 0 & .0 & \\
\hline & BIRADS VI & 0 & .0 & 0 & .0 & 0 & .0 & 0 & .0 & 0 & .0 & 0 & .0 & 0 & .0 & \\
\hline & BIRADS 0 & 0 & .0 & 0 & .0 & 0 & .0 & 1 & 3.4 & 0 & .0 & 0 & .0 & 0 & .0 & \\
\hline
\end{tabular}

\begin{tabular}{|llll|}
\hline & Value & P value \\
\hline Measure of Agreement & Kappa & 0.694 & $<0.001$ \\
\hline
\end{tabular}


Table 6 Agreement between ABUS and mammography BIRADS

\begin{tabular}{|c|c|c|c|c|c|c|c|c|c|c|c|c|c|c|c|c|}
\hline & & \multicolumn{14}{|c|}{ ABUS BIRADS } & \multirow[t]{3}{*}{$\boldsymbol{P}$ value } \\
\hline & & \multicolumn{2}{|c|}{ BIRADS I } & \multicolumn{2}{|c|}{ BIRADS II } & \multicolumn{2}{|c|}{ BIRADS III } & \multicolumn{2}{|c|}{ BIRADS IV } & \multicolumn{2}{|c|}{ BIRADS V } & \multicolumn{2}{|c|}{ BIRADS VI } & \multicolumn{2}{|c|}{ BIRADS 0} & \\
\hline & & Count & $\%$ & Count & $\%$ & Count & $\%$ & Count & $\%$ & Count & $\%$ & Count & $\%$ & Count & $\%$ & \\
\hline \multirow[t]{7}{*}{ Mammography BIRADS } & BIRADS I & 0 & .0 & 0 & .0 & 0 & .0 & 0 & .0 & 0 & .0 & 0 & .0 & 0 & .0 & $<0.001$ \\
\hline & BIRADS II & 0 & .0 & 0 & .0 & 1 & 5.0 & 0 & .0 & 0 & .0 & 0 & .0 & 0 & .0 & \\
\hline & BIRADS III & 0 & .0 & 1 & 50.0 & 14 & 70.0 & 4 & 13.8 & 0 & .0 & 0 & .0 & 0 & .0 & \\
\hline & BIRADS IV & 0 & .0 & 0 & .0 & 0 & .0 & 13 & 44.8 & 3 & 60.0 & 0 & .0 & 0 & .0 & \\
\hline & BIRADS V & 0 & .0 & 0 & .0 & 0 & .0 & 1 & 3.4 & 0 & .0 & 0 & .0 & 0 & .0 & \\
\hline & BIRADS VI & 0 & .0 & 0 & .0 & 0 & .0 & 0 & .0 & 0 & .0 & 0 & .0 & 0 & .0 & \\
\hline & BIRADS 0 & 8 & 100.0 & 1 & 50.0 & 5 & 25.0 & 11 & 37.9 & 2 & 40.0 & 0 & .0 & 0 & .0 & \\
\hline & & & & & & & Value & & P val & & & & & & & \\
\hline & \multicolumn{3}{|c|}{ Measure of Agreement } & & \multicolumn{2}{|c|}{ Kappa } & 0.270 & & \multicolumn{3}{|c|}{$<0.001$} & & & & & \\
\hline
\end{tabular}

as dilated ducts, and a second lesion was retro areolar lesion, interpreted by HHUS as dilated ducts with increased internal vascularity (Table 2).

\section{ABUS}

As regards lesion detectability, it was the highest by ABUS; it could detect 17 lesions missed by mammography, and 2 lesions missed by HHUS.

True positive $=26(53.6 \%)$, false positive $=8(22.2 \%)$, false negative $=2(7.1 \%)$, true negative $=28(77.8 \%)$

As regarding lesions margins in coronal view (which is unique for ABUS), 21 (32.8\%) lesions showed retraction phenomenon (all were malignant 100\%), 21 (32.8\%) lesions showed complete hyperechoic rim (19 (90.5\%) were benign), 14 (21.9\%) lesions showed incomplete (discontinuous) hyperechoic rim (9 (64.3\%) were benign while 5 (35.7\%) were malignant), no masses were detected in $8(12.5 \%)$ cases. Sensitivity of retraction phenomenon for malignancy was $75 \%$, while specificity was $100 \%$. Specificity of hyperechoic rim for benign lesions was $90.5 \%$, while sensitivity for benign lesion detection was 52.8\% (Tables 3, 4, 5 and 6, Figs. 1, 2, and 3).

\section{Discussion}

Breast cancer is the most common malignancy in women from developed and developing countries. Detection and treatment of breast cancer in its earliest possible stage are the ultimate goal. Thus, the role of radiologists in imaging the breast is vital. At present, Xray mammography is the "gold standard" for screening and early detection of breast cancer [8]. Women with dense breast tissue have a high risk of developing breast cancer in a ratio of $15-25 \%$ [9]. ABUS has a promising role in patients with dense breasts in detecting the hidden lesions, as it is a non-operator dependent and it needs less time of interpretation by a radiologist, helping to improve the workflow [10].

Wilczek et al. [12] stated in a study on 1668 asymptomatic women, age 40-74 years, with heterogeneously dense parenchyma (ACR C) or extremely dense breast (ACR D) that the increase in sensitivity of screening for full field digital mammography and 3D ABUS versus FFDM alone was $36.4 \%$. The difference in specificity was $-0.7 \%$.

Giger et al. [13] reported in a study done on 185 asymptomatic women with BI- RADS C or D breast density that the sensitivity was $57.5 \%$ for FFDM alone and $74.1 \%$ for FFDM with ABUS, yielding a statistically significant increase in sensitivity $(P<0.001)$ (relative increase $=29 \%$ ). Overall specificity was $78.1 \%$ for FFDM alone and $76.1 \%$ for FFDM with ABUS $(P=0.496)$.

The Somo insight study [9] preformed on 15,318 women. The sensitivity of mammography alone was 73.2 (95\% CIs $64.9,81$ ) versus $100 \%$ for combined ABUS and mammography. The specificity for mammography alone was 85.4 (95\% CIs 84.9, 86.0) while for combined ABUS and mammography, the specificity was 72.0 (95\% CIs 71.3, 72.7).

A study done on 3418 asymptomatic women with mammographically dense breasts revealed that the sensitivity and specificity of stand-alone digital mammography were $76.00 \%$ (95\% CI, 54.87-90.58\%) and 98.2\% (95\% CI, 97.76-98.59\%) respectively. The positive predictive value was $20.43 \%$ (95\% CI, $12.78-30.05 \%$ ). The 

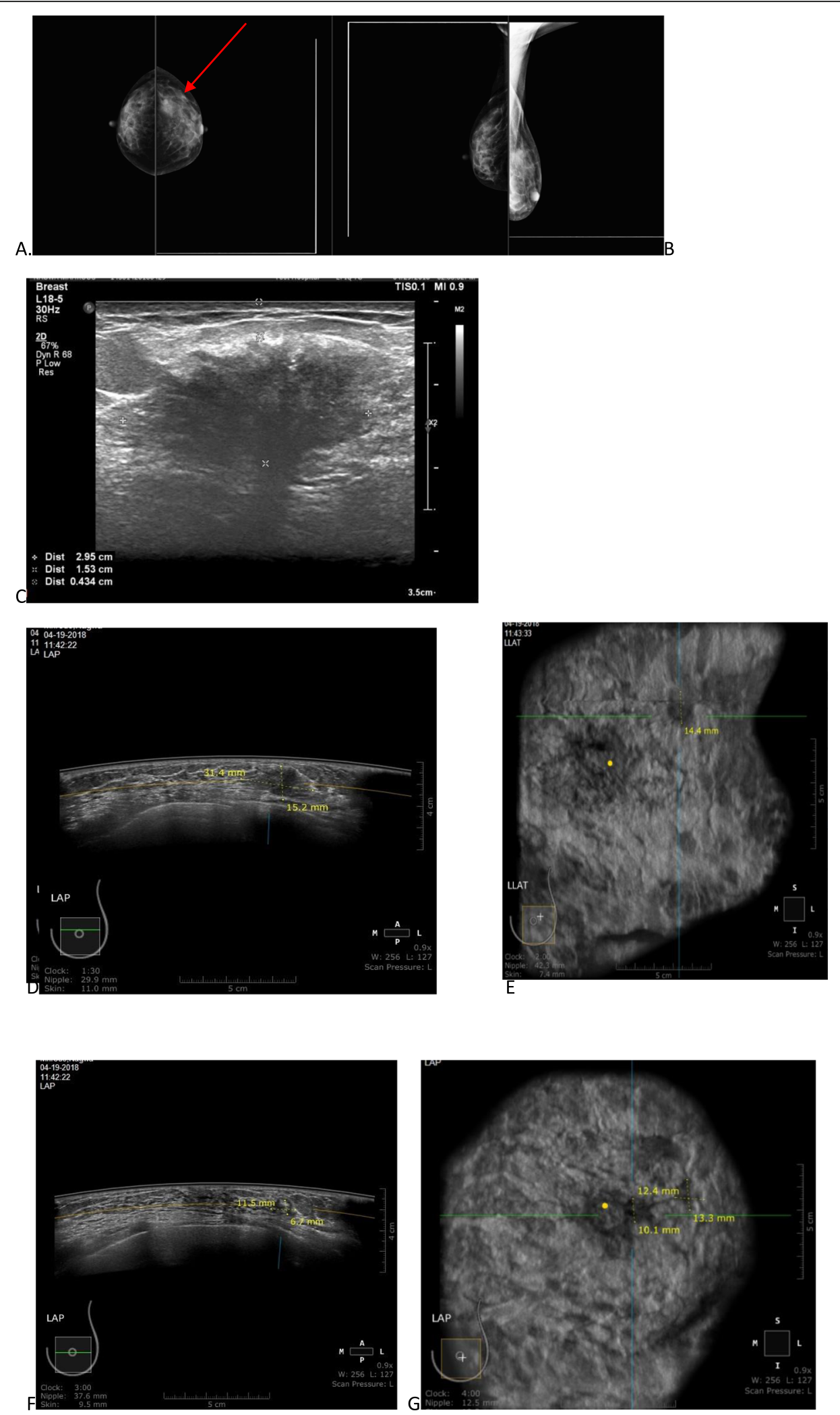

Fig. 1 (See legend on next page.) 
(See figure on previous page.)

Fig. 1 A 53-year-old patient presenting with left breast lump. a, b Mammography revealed ACR D breast with UOQ irregular high densityspeculated mass lesion. Mammography, MLO, and CC views of ACRD revealed suspicious left breast lesion (red arrow); however, proper assessment of the lesion could not be done for further imaging (BIRADS IV). $\mathbf{c}$ HHUS images. HHUS show irregular speculated hypoechoic mass lesion (BIRADS IV). $\mathbf{d}, \mathbf{e}, \mathbf{f}, \mathbf{g}$ ABUS images. Small satellite lesion was detected by ABUS and missed by HHUS, upstaging the disease to multifocal breast cancer. Transverse view of left breast at AP position showing irregular micro lobulated hypoechoic lesion. (BIRADS IV) at 1:30 clock. Coronal view of the left breast in lateral position showing retraction phenomenon at the site of the lesion. Irregular lesion of mixed echogenicity at 3 o'clock likely representing satellite. Coronal view of the left breast in AP position showing retraction phenomenon at the site of the satellite lesions. Biopsy revealed invasive duct carcinoma, grade 2

sensitivity and specificity of ABUS were $97.67 \%$ (95\% CI, 87.67-99.61\%) and 99.70\% (95\% CI, 99.46-99.86\%), respectively. The positive predictive value of ABUS was 80.77\% (95\% CI, 67.46-90.36\%) [14].

In this study, the sensitivity and specificity of standalone digital mammography were 53.6\% (95\% CI, 33.87$72.49 \%$ ) and $91.7 \%$ (95\% CI, 77.53-98.25\%). The positive predictive value was $71.74 \% \quad(95 \% \mathrm{CI}, \quad 62.75$ to 79.27\%). The sensitivity and specificity of ABUS were 92.8\% (95\% CI, 76.50 to $99.12 \%$ ) and $77.78 \%$, (95\% CI, $60.85 \%-89.88 \%)$ respectively. The positive predictive value of ABUS was $76.47 \%$ (95\% CI, 63.62 to $85.80 \%$ ).

To summarize, this study showed the same results compared to the above four studies that ABUS showed an average of $30 \%$ increase in sensitivity in detecting breast malignancy in dense breast compared to digital mammography. As regards specificity, mammography had higher specificity than ABUS in all fore mentioned studies except Wilczek et al. [12], who showed near results of specificity between DM and ABUS but still higher specificity for DM.

Chen et al. [15] stated that there were no significant differences between the ABUS and HHUS in terms of sensitivity $(92.5 \%$ vs. $88.0 \%)$, specificity $(86.2 \%$ vs. $87.5 \%)$, accuracy $(88.1 \%$ vs. $87.2 \%)$, positive predictive value $(74.7 \%$ vs. $75.6 \%)$, and negative predictive value (96.3\% vs. 94.3\%) ( $P, 0.05$ for all).

Choi et al. [16] evaluated a large population of asymptomatic women who were subdivided into two groups (1866 patients for ABUS and 3700 patients for HHUS) and showed that diagnostic accuracy and specificity were significantly higher for ABUS than HHUS (respectively, diagnostic accuracy 97.7 vs. $96.5 \%$ and specificity 97.8 vs. 96.7).

In this study, comparing ABUS versus HHUS as regards sensitivity $(92.8 \%$ vs. $89.3 \%)$, specificity $(77.8 \%$ vs. $88.9 \%)$, accuracy $(82.4 \%$ vs. $89 \%)$, positive predictive value $(76.5 \%$ vs. $86.2 \%)$, and negative predictive value $(93.3 \%$ vs. 91.4\%). In our study, ABUS had higher sensitivity (no significant difference) than HHUS, but HHUS has higher specificity and diagnostic accuracy.

Vourtsis et al. [17] performed a study that included women with breast density category C or D (aged $48.6 \pm 10.8$ years) were recruited. All participants underwent ABUS and HHUS examination; a subcohort of 1665 women also underwent a mammography. The overall agreement between HHUS and ABUS was 99.8\%; kappa $=0.994, P<0.0001$. In this study, the overall agreement between HHUS and ABUS was kappa $=0.694, P<0.0001$, which is lower compared to the above study.

Rella et al. [18] stated that retraction phenomenon (odds ratio [OR], 76.70; 95\% confidence interval [CI], 12.55, 468.70; $P<0.001)$ was the strongest independent predictor for malignant masses.

Chen at al [15]. stated that there were significant differences between the malignant and benign masses with respect to retraction phenomenon and hyperechoic rim in the coronal plane of the ABUS. For retraction phenomenon, both the specificity and positive predictive value of a malignant diagnosis reached $100 \%$, and the accuracy and false-positive rate were $96.8 \%$ and 0 , respectively; for the hyperechoic rim, the specificity, negative predictive value, and accuracy of a benign diagnosis were 92.8\%, 95.3\%, and $95.9 \%$, respectively.

These results are going with this study that retraction phenomenon has a significant relation with malignant pathology $(P$ value $<0.001)$ with $100 \%$ specficity and $75 \%$ sensitivity, while complete hyperechoic rim has significant relation with benign pathology with $(P$ value $<0.001)$ with $90.5 \%$ specificity and $52.8 \%$ sensitivity.

Rella et al. [18] stated that the coronal plane also improves the evaluation of lesion margins; benign tumors are often surrounded by a continuous hyperechoic rim, while breast cancers can present a discontinuous hyperechoic rim. In this study, 14 cases showed incomplete (discontinuous) hyperechoic rim, 9 of them were benign (64\%) while 5 (36\%) were malignant.

Finally, Skane et al. [19] proved that combined mammography and ABUS reading by the same radiologist improved diagnostic performance and resulted in higher observer agreement. Consequently, combined reading mode should be "standard" if ABUS was implemented in screening for women with dense breasts. Prospective studies were necessary before the implementation of ABUS 


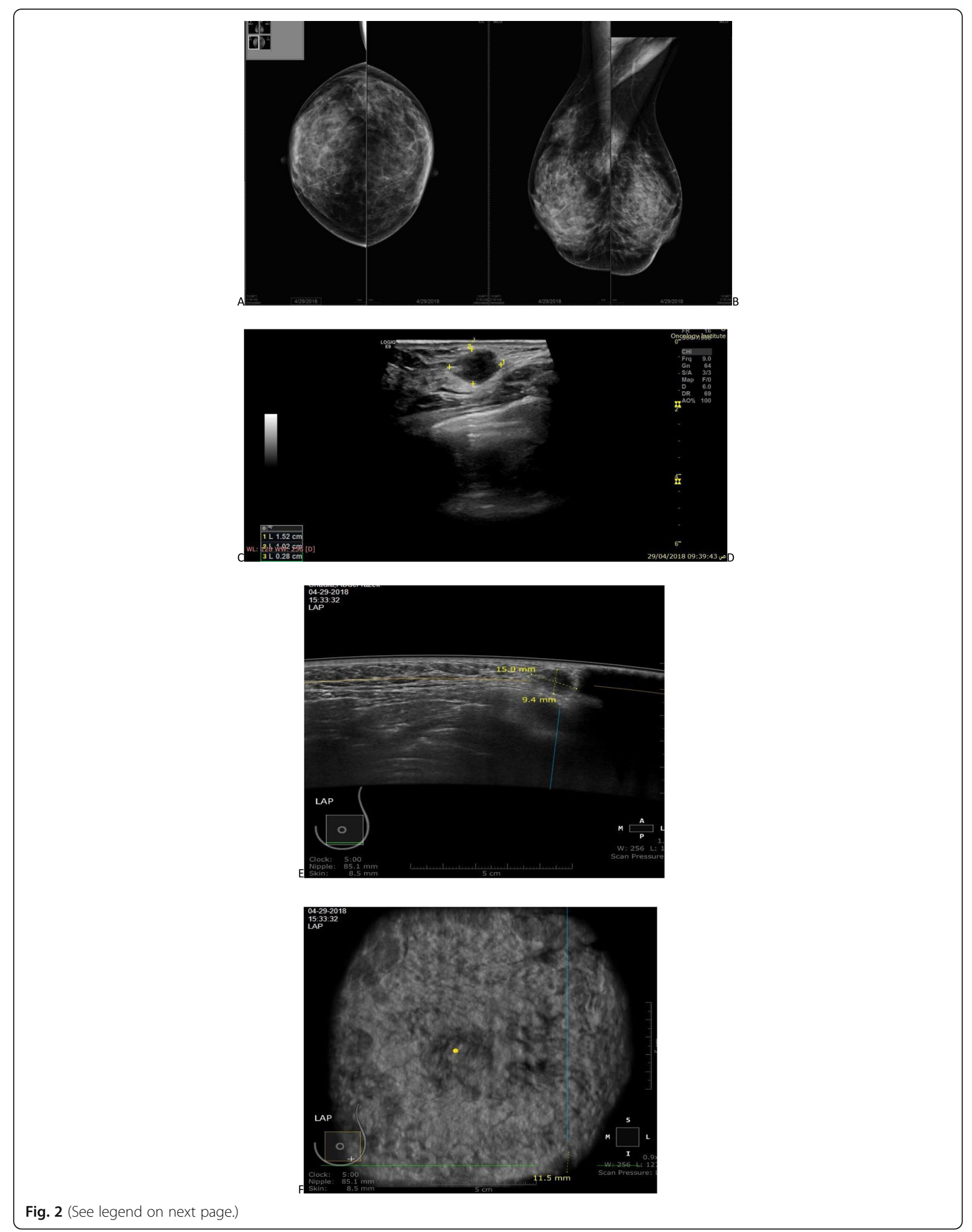


(See figure on previous page.)

Fig. 2 A 34-year-old patient presenting with a left breast lump. a, b Mammography was done and revealed dense breast ( ACR C) (BIRADS 0). c HHUS revealed an irregular microlubolated hypoechoic mass lesion (BIRADS IV). $\mathbf{d}$ ABUS images. Transverse view of the left breast at AP position revealed speculated mass at 5 o'clock (BIRADS IV). e ABUS images. Coronal view of the left breast at lateral position revealed mass with retraction phenomenon at 5 o'clock (BIRADS IV). Pathology revealed IDC grade 2 with DCIS

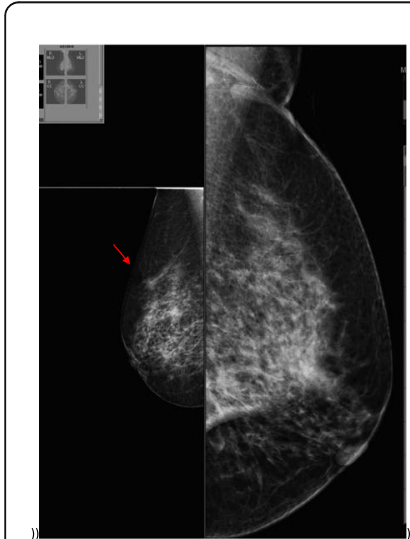

A

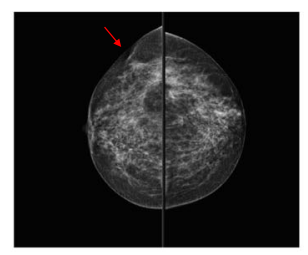

B

Fig. 3 A 47-year-old female patient presenting with bilateral breast lumps. Mammography was done. a, b, c, d Mammography ACR C breasts. MLO view mammography showing ACR C breast with right upper irregular speculated dense lesion. CC view showing outer speculated irregular dense mass lesion for further evaluation (BIRADS IV). e, $\mathbf{f}, \mathbf{g}, \mathbf{h}$ HHUS images. HHUS of left breast showing irregular hypoechoic speculated mass lesion at 6 o'clock. Satellite at the left breast denoting multifocal breast cancer. HHUS of left axilla with pathological LN showing cortical thickening. HHUS of right breast showing irregular hypoechoic speculated mass lesion at 10 o'clock. F. satellite at the right breast denoting multifocal breast cancer. $\mathbf{i}, \mathbf{j}, \mathbf{k}$, I ABUS images. Coronal view of the left breast in AP position showing retraction phenomenon.at the site of the lesion. Transverse view of the left breast in AP position showing irregular hypoechoic speculated mass lesion at 5 o'clock. Transverse view of the right breast in lateral position showing irregular hypoechoic speculated non-parallel mass lesion. Coronal view of the right breast in lateral position showing retraction phenomenon at the site of the lesion. Pathology revealed left breast lesion: ILC. Right breast lesion: IDC could be recommended in population-based screening. This study also went finally with same recommendation as mammography still could detect DCIS before IDC development as per our knowledge; further research is also recommended for this point.

The potential role of ABUS in the follow-up of benign lesions was supported by its considerable reliability in the recording of lesion location, distance from the nipple, and lesion size, these features suggested potential use in the follow-up of benign lesions as per Chang et al. [20].

\section{Conclusion}

The sensitivity of ABUS in detecting breast lesions is much higher than mammography in dense breast, while the DM has higher specificity. We recommend the implementation of both DM and ABUS to get benefit of DM specificity as well as ABUS sensitivity.

\section{Abbreviations}

NPV: Negative predictive value; PPV: Positive predictive value; ABUS: Automated breast US; DM: Digital mammography; HHUS: Handheld ultrasound; FFDM: Fullfield digital mammography

\section{Acknowledgements}

First and foremost, thanks to Allah, the most beneficial and most merciful. It is but for His mercy that we can put through in life.

\section{Authors' contributions}

AM analyzed and interpreted the patient data regarding the breast lesions. EA performed the ultrasound examination of the breast, and was a major contributor in writing the manuscript. NA participated in writing the final manuscript. All authors read and approved the final manuscript.

Funding

Not applicable. 


\section{Availability of data and materials}

The data sets used and/or analyzed during the current study are available from the corresponding author on reasonable request.

\section{Ethics approval and consent to participate}

The study is a prospective study that was reviewed by the ethics committee of Radiology Departments and was approved by the review board that is related to our University. Patients included gave informed written consent to use their data in research work. No applicable reference number.

\section{Consent for publication}

All the patients included in this research gave written informed consent to publish the data contained within this study.

\section{Competing interests}

The authors declare that they have no competing interests.

Received: 3 December 2019 Accepted: 17 March 2020

Published online: 27 August 2020

\section{References}

1. Torre LA, Siegel RL, Ward EM, Jemal A (2016) Global cancer incidence and mortality rates and trends - an update. Cancer Epidemiol Biomarkers Prev 25(1):16-27

2. Etzioni R, Urban N, Ramsey S, Mclntosh M, Schwartz S, Reid B et al (2003) Early detection: the case for early detection. Nat Rev Cancer 3(4):243

3. Hofvind S, Ursin G, Tretli S, Sebuodegard S, Moller B (2013) Breast cancer mortality in participants of the Norwegian breast cancer screening program. Cancer 119:3106-3112

4. Tabár L, Vitak B, Chen THH, Yen AMF, Cohen A, Tot \& Fohlin, H (2011) Swedish two-county trial: impact of mammographic screening on breast cancer mortality during 3 decades. Radiology 260(3):658-663

5. Kuhl CK, Schrading S, Leutner CC, Morakkabati-Spitz N, Wardelmann E, Fimmers R et al (2005) Mammography, breast ultrasound, and magnetic resonance imaging for surveillance of women at high familial risk for breast cancer. J Clin Oncol 23(33):8469-8476

6. Boyd NF, Guo H, Martin LJ, Sun L, Stone J, Fishell E et al (2007) Mammographic density and the risk and detection of breast cancer. N Engl J Med 356(3):227-236

7. Yaghjyan L, Colditz GA, Collins LC, Schnitt SJ, Rosner B, Vachon C et al (2011) Mammographic breast density and subsequent risk of breast cancer in postmenopausal women according to tumor characteristics. J Natl Cancer Inst 103:1179-1189

8. Coughlin SS, Ekwueme DU (2009) Breast cancer as a global health concern. Cancer Epidemiol 33(5):315-318

9. Brem RF, Tabár L, Duffy SW et al (2015) Assessing improvement in detection of breast cancer with three-dimensional automated breast US in women with dense breast tissue: the Somolnsight study. Radiology 274:663-673

10. Shin HJ, Kim HH, Cha JH (2015) Current status of automated breast ultrasonography. Ultrasonography 34(3):165

11. Kaplan SS (2014) Automated whole breast ultrasound. Radiol Clin North Am. 52:539-546

12. Wilczek B, Wilczek HE, Rasouliyan L, Leifland K (2016) Adding 3D automated breast ultrasound to mammography screening in women with heterogeneously and extremely dense breasts: report from a hospitalbased, high-volume, single-center breast cancer screening program. Eur J Radiol 85:1554-1563

13. Giger ML, Inciardi MF, Edwards A et al (2016) Automated breast ultrasound in breast cancer screening of women with dense breasts: reader study of mammography-negative and mammography-positive cancers. AJR Am J Roentgenol 206:1-10

14. Giuliano V, Giuliano C (2013) Improved breast cancer detection in asymptomatic women using 3D-automated breast ultrasound in mammographically dense breasts. Clin Imaging 37(3):480-486

15. Chen L, Chen Y, Diao XH, Fang L, Pang Y, Cheng AQ et al (2013) Comparative study of automated breast 3-D ultrasound and handheld Bmode ultrasound for differentiation of benign and malignant breast masses. Ultrasound Med Biol 39(10):1735-1742

16. Choi WJ, Cha JH, Kim HH et al (2014) Comparison of automated breast volume scanning and hand-held ultrasound in the detection of breast cancer: an analysis of 5,566 patients evaluations. Asian Pac J Cancer Prev 15: 9101-9105

17. Vourtsis A, Kachulis A (2018) The performance of 3D ABUS versus HHUS in the visualisation and BI-RADS characterisation of breast lesions in a large cohort of 1,886 women. Eur Radiol 28(2):592-601

18. Rella R, Belli P, Giuliani M, Bufi E, Carlino G, Rinaldi P, Manfredi R (2018) Automated breast ultrasonography (ABUS) in the screening and diagnostic setting: indications and practical use. Acad Radiol 25(11):1457-1470

19. Skaane P, Gullien R, Eben EB, Sandhaug M, Schulz-Wendtland R, Stoeblen F (2015) Interpretation of automated breast ultrasound (ABUS) with and without knowledge of mammography: a reader performance study. Acta Radiologica 56(4):404-412

20. Chang JM, Cha JH, Park JS, Kim SJ, Moon WK (2015) Auto- mated breast ultrasound system (ABUS): reproducibility of mass localization, size measurement, and characterization on serial examinations. Acta Radiol 56: $1163-1170$

\section{Publisher's Note}

Springer Nature remains neutral with regard to jurisdictional claims in published maps and institutional affiliations.

\section{Submit your manuscript to a SpringerOpen ${ }^{\circ}$ journal and benefit from:}

- Convenient online submission

- Rigorous peer review

- Open access: articles freely available online

High visibility within the field

- Retaining the copyright to your article

Submit your next manuscript at $\boldsymbol{\nabla}$ springeropen.com 